INPLASY

PROTOCOL

To cite: Zhang et al. Moxibustion for treating intradialytic hypotension: A protocol for systematic review and meta-analysis. Inplasy protocol 2020120122. doi: 10.37766/inplasy2020.12.0122

Received: 24 December 2020

Published: 24 December 2020

Corresponding author: Yong Liu

413320631@qq.com

Author Affiliation: Chengdu University of Traditional Chinese Medicine, Chengdu, Sichuan, China

Support: SMA:

No.2017SHD012.

Review Stage at time of this submission: The review has not yet started.

Conflicts of interest: None.

\section{Moxibustion for treating intradialytic hypotension: A protocol for systematic review and meta-analysis}

Zhang, Z1; Hu, L2; Xia, W3; Liu, S4.

Review question / Objective: The aim of this overview is to synthesize and assess the reliability of evidence generated from these systematic reviews of the effectiveness of moxibusition for intradialytic hypotension.

Condition being studied: Intradialytic hypotension(IDH) has been reported in $\mathbf{2 0 - 3 0 \%}$ of patients treated with maintenance haemodialysis(HD). The symptoms associated with this clinical problem, such as nausea, dizziness andcramps, can have a negative impact on patients'quality of life and tolerability to dialysis. In traditional Chinese medicine(TCM) theory, symptomatic IDH is aetiologically caused by the derangement of Qi-blood and subsequent prostration of Yang-Qi in the human body during the rapid fluid removal of HD. Accumulating evidenceshows that applying moxibustion to specific acupoints has a theapeutic effect on clinical symptoms and quality of life in patients with HD. Nevertheless, the evidence for the effectiveness of moxibustion as the treatment for IDH has not been thoroughly evaluated yet. Therefore, we conducted this overview of moxibusition as intervention for IDH patients, critically appraised and synthesized the results from these SRs in order to provide more reliable evidence-based medical references for clinical practitioners and researchers.

INPLASY registration number: This protocol was registered with the International Platform of Registered Systematic Review and Meta-Analysis Protocols (INPLASY) on 24 December 2020 and was last updated on 24 December 2020 (registration number INPLASY2020120122).

\section{INTRODUCTION}

Review question / Objective: The aim of this overview is to synthesize and assess the reliability of evidence generated from these systematic reviews of the effectiveness of moxibusition for intradialytic hypotension. 
Condition being studied: Intradialytic hypotension(IDH) has been reported in $20-30 \%$ of patients treated with maintenance haemodialysis(HD). The symptoms associated with this clinical problem, such as nausea, dizziness andcramps, can have a negative impact on patients'quality of life and tolerability to dialysis. In traditional Chinese medicine(TCM) theory, symptomatic IDH is aetiologically caused by the derangement of Qi-blood and subsequent prostration of Yang-Qi in the human body during the rapid fluid removal of HD. Accumulating evidenceshows that applying moxibustion to specific acupoints has a theapeutic effect on clinical symptoms and quality of life in patients with HD. Nevertheless, the evidence for the effectiveness of moxibustion as the treatment for IDH has not been thoroughly evaluated yet. Therefore, we conducted this overview of moxibusition as intervention for IDH patients, critically appraised and synthesized the results from these SRs in order to provide more reliable evidencebased medical references for clinical practitioners and researchers.

\section{METHODS}

Search strategy: The electronic database, including PubMed, Cochrane Library, EMBASE, the China National Knowledge Infrastructure(CNKI), China Biology Medicine disc(CBM), VIP database and Wangfang database, were thoroughly retrieved from inception to January 1st, 2022, without language restrictions.

Participant or population: Patients include those diagnosed with IDH, regardless of gender, age, nationality or race.

Intervention: Systematic Reviews(SRs) that involved any form of moxibustion(e.g., direct moxibustion, indirect moxibustion, heat-sensitive moxibustion, moxa burner moxibustion, warm needling, crude drug moxibustion, or natural moxibustion) as the sole treatment or as a part of a combination therapy with another intervention (e.g., conventional drugs) will be included.
Comparator: There is no limit to the treatment of the control group, including no treatment, or placebo, or anycontrol considered for comparison in the individual system review.

Study designs to be included: SRs of randomized controlled trials(RCTs) were included, in which moxibistion was utilized as the treatment for IDH.

Eligibility criteria: The SRs of moxibustion for IDH met the inclusion criteria as following were included. 1.Types of studies: SRs of randomized controlled trials(RCTs) were included, in which moxibistion was utilized as the treatment for IDH. 2.Types of participants: Patients include those diagnosed with IDH, regardless of gender, age, nationality or race. 3.Types of interventions: SRs that involved any form of moxibustion (e.g., direct moxibustion, indirect moxibustion, heat-sensitive moxibustion, moxa burner moxibustion, warm needling, crude drug moxibustion, or natural moxibustion) as the sole treatment or as a part of a combination therapy with another intervention (e.g., conventional drugs) will be included. 4.Type of comparator: There is no limit to the treatment of the control group, including no treatment, or placebo, or any control considered for comparison in the individual system review. 5.Types of outcome measures: The main outcome measure will be the percentage of target UF.

Information sources: We will use computers to retrieve all RCTs of IDH on PubMed, Cochrane Library, EMBASE, CNKI, Wanfang, CBM and VIP databases. At the same time, we will supplement it by searching relevant literature manually.

Main outcome(s): The aetiology of IDH is multifactorial. The main factor is rapid removal of intravascular volume by ultrafiltration(UF) and the subsequent imbalance between UF and the plasma refilling rate. The main outcome measure will be the percentage of target UF. Target UF volume will be the difference between the predialysis weight and the dry weight. Actual UF volume will be the difference 
between the predialysis and postdialysis weights.

Quality assessment / Risk of bias analysis: To assess the risk of bias for all included studies, Cochrane Collaboration's bias risk tool will be used by two independent review authors to assess the following areas: random sequence generation, allocation concealment, blindness to participants, people, and results, incomplete outcome data, optional reporting, and other biases. Any discrepancies in the deviation risk assessment will be resolved through discussion. Ultimately, the quality of the studies will be divided into three levels: low risk of bias, high risk of bias, and unclear risk of bias.

Strategy of data synthesis: Strategy of data synthesis: All analyses will be conducted by using RevMan software (V5.3), We will select fixed effects model or random effects model to merge the outcome indicators in accordance with the results of heterogeneity test. The fixed effects model will be applied for data synthesis of low heterogeneity $(12<50 \%)$ while the random effects model will be conducted if the heterogeneity is significant $(12 \geq 50 \%)$. It is considered that differences are statistically significant if the results of $Z$ test show that $P$ value is less than 0.05 , and the $95 \% \mathrm{CI}$ does not contain 0 (for continuous variables) or the $95 \% \mathrm{Cl}$ does not contain 1 (for dichotomous variables).

Subgroup analysis: If the necessary data are available, subgroup analysis will be carried out according to different factors as follows:1.Control interventions(e.g., sham/ placebo moxibustion, notreatment, other TCM treatment or non-TCM treatment). 2.Type of moxibustion(e.g., heat-sensitive moxibustion, thunderfire miraculous moxa roll, warm needling moxibustion, suspended moxibustion or mild moxibustion).

Sensibility analysis: If the heterogeneity of the included literature is significant, in order to ensure the credibility of there search results, we will conduct sensitivity analysis by excluding each included study separately, so as to improve the research quality.

Language: Chinese and English.

Country(ies) involved: China.

Keywords: moxibustion, intradialytic hypotension, systematic review, protocol.

Contributions of each author:

Author 1 - Ziqu Zhang.

Author 2 - Lishu Hu.

Author 3 - Wengang Xia.

Author 4 - Shuqin Liu. 